\title{
Papillary carcinoma of the breast lacks evidence of $R E T$ rearrangements despite morphological similarities to papillary thyroid carcinoma
}

\author{
Omar Hameed ${ }^{1}$, Arie Perry ${ }^{2}$, Ruma Banerjee ${ }^{2}$, Xiaopei Zhu² and John D Pfeifer ${ }^{2}$ \\ ${ }^{1}$ Departments of Pathology and Surgery and the Comprehensive Cancer Center, University of Alabama at \\ Birmingham, Birmingham, AL, USA and ${ }^{2}$ Department of Pathology and Immunology, Washington University \\ School of Medicine, St Louis, MO, USA
}

\begin{abstract}
Rare breast neoplasms resembling the tall-cell variant of papillary thyroid carcinoma have been reported. In addition, papillary carcinoma of the breast occasionally displays nuclear features reminiscent of papillary thyroid carcinoma. In this study, we evaluated 33 intraductal/intracystic papillary carcinomas of the breast for the presence and extent of nuclear overlap, grooves, clearing, and inclusions, as well as features of the tall-cell or columnar-cell variants of papillary thyroid carcinoma. RET rearrangements were assessed in a subset of these cases. Paired probes localizing to the centromeric and telomeric ends of the RET gene on chromosome 10 were used for FISH using a break-apart approach. Single round and nested PCR was performed to detect RET/ PTC1, RET/PTC2, RET/PTC3 and ELKS-RET fusion transcripts. Nuclear overlap, grooves, stratification, and clearing were identified in $24(73 \%), 14(42 \%), 11(33 \%)$, and $9(27 \%)$ cases respectively, whereas nuclear inclusions and 'tall-cell' features were each seen in only one (3\%) and two $(6 \%)$ cases, respectively. Four of 19 tested cases displayed split FISH signals in a low percentage of cells and were considered borderline for RET rearrangement. All 19 tested cases with amplifiable RNA were negative for the four RET fusion transcripts evaluated by RT-PCR. Although papillary carcinomas of breast often display one or more cytoarchitectural features of papillary thyroid carcinoma, there is no evidence that RET rearrangements are involved. Modern Pathology (2009) 22, 1236-1242; doi:10.1038/modpathol.2009.91; published online 19 June 2009
\end{abstract}

Keywords: papillary carcinoma; breast; $R E T$

Excluding lesions partly involving benign papillomas, malignant papillary lesions of the breast include papillary ductal carcinoma in situ, intracystic (encapsulated) papillary carcinoma, and solid papillary carcinoma, all of which may or may not be associated with unequivocal invasive carcinoma. ${ }^{1,2}$ All of these lesions are characterized by an expansile epithelial proliferation composed of fibrovascular cores covered by rows of epithelial cells with no or minimal myoepithelial cell participation. Although the epithelial component can have a solid, cribriform or micropapillary growth pattern, it more

Correspondence: Dr O Hameed, MBChB, Division of Anatomic Pathology, Departments of Pathology and Surgery, University of Alabama at Birmingham, 619 19th St South, NP 3550, Birmingham, AL 35294-6823, USA.

E-mail: ohameed@uab.edu

Presented in part at the 98th Annual Meeting of the United States and Canadian Academy of Pathology, Boston, MA, March, 2009 Received 01 April 2009; revised 20 April 2009; accepted 27 April 2009; published online 19 June 2009 frequently consists of one or more layers of columnar cells with a variable degree of nuclear stratification. ${ }^{1-3}$ This latter appearance is not unique to these neoplasms but is also shared with other papillary carcinomas including, among others, papillary thyroid carcinoma, which characteristically displays nuclear overlap and stratification.

In 2003, Eusebi et $a l^{4}$ described five breast neoplasms composed of columnar to cuboidal eosinophilic cells with grooved nuclei, variable nuclear clearing and occasional intranuclear inclusions. Given their resemblance to one of the variants of papillary thyroid carcinoma (which was excluded by both clinical and immunohistochemical means), such cases were referred to as 'breast tumor resembling the tall-cell variant of papillary thyroid carcinoma.' Since then, five additional cases have been reported with similar clinical, morphological, and immunohistochemical findings. ${ }^{5,6}$ In addition to displaying nuclear features characteristic of papillary thyroid carcinoma, all 10 reported cases 
had unequivocal papillary areas, and most displayed evidence of an in situ component and/or had foci surrounded at the periphery by a myoepithelial cell layer as determined morphologically or by immunohistochemistry. ${ }^{4-6}$ These findings are similar to papillary carcinomas of the breast (with or without invasion), which can also have residual myoepithelial cells at the periphery, ${ }^{1,2}$ suggesting that some of these cases may represent papillary carcinoma of the breast, albeit with prominent cytoarchitectural features of papillary thyroid carcinoma. If this were the case, one might expect that such features need not be limited to cases of 'breast tumor resembling the tall-cell variant of papillary thyroid carcinoma', but may be common to papillary carcinomas of the breast in general.

Accordingly, the goal of this study was first to document the prevalence of papillary thyroid carcinoma-like cytoarchitectural features ${ }^{7}$ (including the tall-cell and columnar-cell variants) in breast papillary carcinomas and second, to determine the prevalence of papillary thyroid carcinoma-like RET tyrosine kinase proto-oncogene chromosomal rearrangements in these tumors. ${ }^{8,9}$

\section{Materials and methods}

\section{Case Selection and Histological Review}

Thirty-three consecutive papillary carcinomas of the breast with or without associated invasive carcinoma, diagnosed over a 2-year period at the Lauren V Ackerman Laboratory of Surgical Pathology, Washington University Medical Center were histologically reviewed to confirm the diagnosis and evaluate for the presence or absence of papillary thyroid carcinoma-like morphological features, including nuclear overlap, grooves, clearing, and inclusions, ${ }^{7,10}$ tall eosinophilic cells with a height at least two times their width, as seen in the tall-cell variant, ${ }^{10,11}$ and/or prominent stratification of elongated nuclei within the cores (with or without cytoplasmic clearing or vacuolization) as seen in the columnar cell variant. ${ }^{10}$ Each of these features was scored as 0 (absent), $1+$ (present in $<5 \%$ of examined high power fields of the lesion), $2+(5-25 \%$ of examined high power fields of the lesion) and $3+(>25 \%$ of examined high power fields of the lesion).

\section{Molecular Techniques}

Fluorescent in situ hybridization (FISH)

Dual color FISH experiments utilizing a break-apart approach to identify rearrangements of the RET locus were performed as previously described. ${ }^{12}$ Briefly, thin sections (5-6 microns) from a previously selected formalin-fixed paraffin-embedded block for each tumor were mounted onto poly Llysine coated slides. Following deparaffinization, the sections were subjected to target retrieval utilizing 'steam cooking' in citrate buffer for $20 \mathrm{~min}$, followed by a $20 \mathrm{~min}$ cool down period and a 5 min wash with distilled water. After pepsin digestion at $37^{\circ} \mathrm{C}$ for $30 \mathrm{~min}$ and a subsequent wash in $2 \times$ SSC, the slides were air dried. Two fluorochrome-labeled bacterial artificial chromosome-derived probes were utilized, one centromeric to RET (RP11-379D20) and another telomeric to it (RP11-348I23) (detailed information available at http://www.genome.ucsc.edu/cgi-bin/hgGateway). Dual target hybridizations were performed in DenHyB buffer (Insitus Biotechnologies, Albuquerque, NM, USA). The hybridization mix $(10 \mu \mathrm{l})$ was applied to the sections, followed by simultaneous denaturing of probe and target at $90^{\circ} \mathrm{C}$ for $13 \mathrm{~min}$. Hybridization was performed overnight at $37^{\circ}$ in a humidified chamber. Post-hybridization washes in $50 \%$ formamide $/ 1 \times$ SSC $(5 \mathrm{~min})$ and $2 \times$ SSC ( $5 \mathrm{~min}$ ) were performed at room temperature, and the slides were again air-dried. Nuclei were then counter-stained with DAPI $(0.5 \mu \mathrm{l} / \mathrm{ml})$ and the sections were examined using an Olympus BX60 Fluorescent Microscope with appropriate filters (Olympus, Melville, NY, USA).

A minimum of 100 non-overlapping intact nuclei were assessed for the presence of splitting of a normally fused yellow or red-green signal; the interphase FISH scoring was performed blinded to the tissue diagnosis. Splitting was defined as green and red signals further than one signal diameter apart. Both a papillary thyroid carcinoma cell line previously identified as harboring a RET/PTC1 rearrangement ${ }^{13}$ and two cases of papillary thyroid carcinoma previously found to show RET rearrangement by FISH were used as positive controls, whereas adjacent non-neoplastic breast and thyroid tissues were used as internal negative controls. A split FISH signal was seen in $1-3 \%$ of the nuclei in the negative control tissues suggesting a cutoff of $5 \%$ split signals for positive results (median +3 s.d.).

\section{RNA extraction}

Four $10 \mu \mathrm{m}$ sections of tumor were deparaffinized using xylene and ethanol washes, and digested in buffer (Tris pH 7.5, $20 \mathrm{mM}$ EDTA, 1\% SDS) containing $25 \mathrm{mg}$ proteinase $\mathrm{K}$. Total RNA was then extracted from tumor tissue using a modified guanidine isothiocyanate method employing Trizol (Gibco/BRL, Gaithersburg, MD, USA) as previously described, ${ }^{14}$ and treated with DNAse I before reverse transcription.

\section{RT-PCR}

RNA samples were reverse transcribed using Mulv reverse transcriptase and the Gene Amp RNA-PCR kit (Perkin-Elmer, Foster City, CA, USA). Single round PCR was performed for RET/PTC and ELKS$R E T$ fusion transcripts using previously published primers $^{15,16}$ (Table 1) in $50 \mu \mathrm{l}$ final volume as follows: after initial denaturation at $95^{\circ} \mathrm{C}$ for $5 \mathrm{~min}$, 35 cycles of amplification were performed with 
Table 1 PCR primers used in the study for the evaluation of the RET oncogene

\begin{tabular}{|c|c|c|c|}
\hline Primer pair & Primer name (gene) & Primer sequence & Amplicon size \\
\hline \multirow[t]{2}{*}{ RET/PTC1 outer pair } & $1(H 4)$ & 5'-AGATAGAGCTGGAGACCTAC-3' & $379 \mathrm{bp}$ \\
\hline & $10(R E T$ exon 14$)$ & 5'-GAGCCGTATTTGGCGTACTC-3' & \\
\hline \multirow{2}{*}{$R E T / P T C 1$ inner pair } & $2(H 4)$ & 5'-GCTGGAGACCTACAAACTGA-3' & $165 \mathrm{bp}$ \\
\hline & $12(R E T$ exon 12$)$ & 5'-CGTTGCCTTGACCACTTTTC-3' & \\
\hline \multirow[t]{2}{*}{ RET/PTC2 outer pair } & $4(R I \alpha)$ & $5^{\prime}$-AGGGAGCTTTGGAGAACTTG-3' & $414 \mathrm{bp}$ \\
\hline & $10($ RET exon 14$)$ & $5^{\prime}$-GAGCCGTATTTGGCGTACTC- $3^{\prime}$ & \\
\hline \multirow[t]{2}{*}{ RET/PTC2 inner pair } & $5(R I \alpha)$ & 5'-GCTTTGGAGAACTTGCTTTGATTT-3' & $202 \mathrm{bp}$ \\
\hline & $12(R E T$ exon 12$)$ & 5'-CGTTGCCTTGACCACTTTTC-3' & \\
\hline \multirow{2}{*}{ RET/PTC3 outer pair } & $7(E L E 1)$ & $5^{\prime}$-CATGCCAGAGCAGAAGTCA-3' & $453 \mathrm{bp}$ \\
\hline & $10(R E T$ exon 14$)$ & 5'-GAGCCGTATTTGGCGTACTC-3' & \\
\hline \multirow[t]{2}{*}{ RET/PTC3 inner pair } & $8(E L E 1)$ & 5'-AAGCAAACCTGCCAGTGG-3’ & 242 bp \\
\hline & 11 (RET exon 12) & 5'-CTTTCAGCATCTTCACGG-3' & \\
\hline \multirow[t]{2}{*}{ ELKS-RET outer pair } & $14(E L K S)$ & 5'-CACTGACACTGCCTTGACA-3' & $476 \mathrm{bp}$ \\
\hline & 11 (RET exon 12) & $5^{\prime}$-CTTTCAGCATCTTCACGG-3' & \\
\hline \multirow{2}{*}{$E L K S-R E T$ inner pair } & $15(E L K S)$ & 5'-TCTTCTCTGGCATCCTCAGG-3' & $259 \mathrm{bp}$ \\
\hline & 11 (RET exon 12) & 5'-CTTTCAGCATCTTCACGG-3' & \\
\hline \multirow[t]{2}{*}{$\beta 2$-microglobulin } & Sense & $5^{\prime}$-ACCCCCACTGAAAAAGATGA- $3^{\prime}$ & $114 \mathrm{bp}$ \\
\hline & Antisense & 5'-ATCTTCAAACCTCCATGATG-3' & \\
\hline
\end{tabular}

denaturation at $94^{\circ} \mathrm{C}$ for $1 \mathrm{~min}$, annealing at $55^{\circ} \mathrm{C}$ for $30 \mathrm{~s}$, and extension at $72^{\circ} \mathrm{C}$ for $1 \mathrm{~min}$. For nested PCR, an initial round of amplification (using the 'outer pair' primer sets from Table 1 and the same cycle parameters as for single round PCR) was followed by the nested round of amplification (using the 'inner pair' primer sets and the same cycle parameters) in which $5 \mu$ l of the initial PCR product was added as the template. PCR products were visualized by ethidium bromide staining after separation by $2 \%$ agarose gel electrophoresis. In each experiment, RNA from the papillary thyroid carcinoma cell line previously identified as harboring a RET/PTC1 rearrangement was assayed in parallel as the positive control. A no input DNA (reagent only) sample and a sham reverse transcriptase-treated sample were assayed in parallel as negative controls. To verify the presence of intact RNA and amplifiable cDNA, each reverse transcriptase reaction product was also assayed with primers for the housekeeping gene encoding $\beta 2$-microglobulin (Table 1).

\section{Photography}

FISH slides were digitally photographed using a high-resolution black and white COHU CCD camera, with a Z-stack motor programmed to capture images at sequential DAPI (one level), FITC (five levels), and rhodamine (five levels) filter settings. Reconstruction into a single superimposed image with blue, green, and red pseudocolors was accomplished using a CytoVisionTM basic workstation (Applied Imaging, Santa Clara, CA, USA).

\section{Regulatory Approval}

The Human Studies Committee at Washington University Medical Center approved testing of existing pathological specimens for this study.

\section{Results}

\section{Clinical Features and Pathological Diagnosis}

The 33 cases included material obtained from 6-needle core and 27 excisional biopsies performed on 28 women and one man. The age of the patients ranged from 41 to 87 years, with mean and median ages of 67.2 and 71 years, respectively. Nineteen specimens were from the left breast and 14 were from the right. The cases included two cases of papillary ductal carcinoma in situ (including one associated with invasive ductal carcinoma, not otherwise specified), 25 intracystic/encapsulated papillary carcinomas (including seven associated with invasive ductal carcinoma, not otherwise specified), and six solid papillary carcinomas (including two associated with invasive mucinous carcinoma).

\section{Cytoarchitectural Features of Papillary Thyroid Carcinoma}

Nuclear overlap, nuclear grooves, stratification, and nuclear clearing were at least focally identified in $24(73 \%), 14(42 \%), 11(33 \%)$, and $9(27 \%)$ cases respectively, whereas nuclear inclusions and tallcell' features were each seen in only one (3\%) and two (6\%) cases, respectively (Table 2). Representative cases are displayed in Figure 1. Seven (21\%) cases did not show any cytoarchitectural features of papillary thyroid carcinoma, whereas $11(33 \%)$, $4(12 \%), 5(15 \%)$ and $6(18 \%)$ cases simultaneously showed one, two, three, or four of these features, respectively. Moreover, these cytoarchitectural features were identified in $23(92 \%)$ of the 25 intracystic/encapsulated papillary carcinomas but in only one $(17 \%)$ of the six solid papillary carcinomas $(P=0.0007$; Fisher's exact test). This 
latter single case only focally displayed nuclear grooves.

\section{FISH and RT-PCR Findings}

Four of 19 tested cases displayed split FISH signals in a low percentage of cells (range: 9-11\%)

Table 2 The prevalence of cytoarchitectural features commonly seen in papillary thyroid carcinoma in 33 papillary carcinomas of the breast

\begin{tabular}{lrrrr}
\hline \multirow{2}{*}{ Histological feature } & \multicolumn{4}{c}{ Number of cases } \\
\cline { 2 - 5 } & $1+$ & $2+$ & $3+$ & Total (\%) \\
\cline { 2 - 5 } & 13 & 8 & 3 & $24(73)$ \\
Nuclear overlap & 12 & 1 & 1 & $14(42)$ \\
Nuclear grooves & 1 & 2 & 8 & $11(33)$ \\
Stratification & 6 & 2 & 1 & $9(27)$ \\
Nuclear clearing & 0 & 1 & 0 & $1(3)$ \\
Nuclear inclusions & 0 & 1 & 1 & $2(6)$ \\
\hline
\end{tabular}

(Figure 2). Given that this percentage was much lower than that seen in the positive controls cases ( $>80 \%$ in the papillary thyroid carcinoma cell line; 40 and $50 \%$ in the papillary thyroid carcinoma patient specimens), these cases were considered borderline for RET rearrangement. These four cases, as well as another 15 cases with amplifiable RNA (out of 22 tested cases) were all negative for the four RET fusion transcripts evaluated by RT-PCR (Figure 3).

\section{Discussion}

In this study, we have shown that the presence of cytoarchitectural features characteristic of papillary thyroid carcinoma are not just limited to the reported cases of so-called "breast tumor resembling the tall-cell variant of papillary thyroid carcinoma,' as papillary carcinomas of the breast also display such features to a variable extent. Despite this morphologic resemblance, however, the RET gene
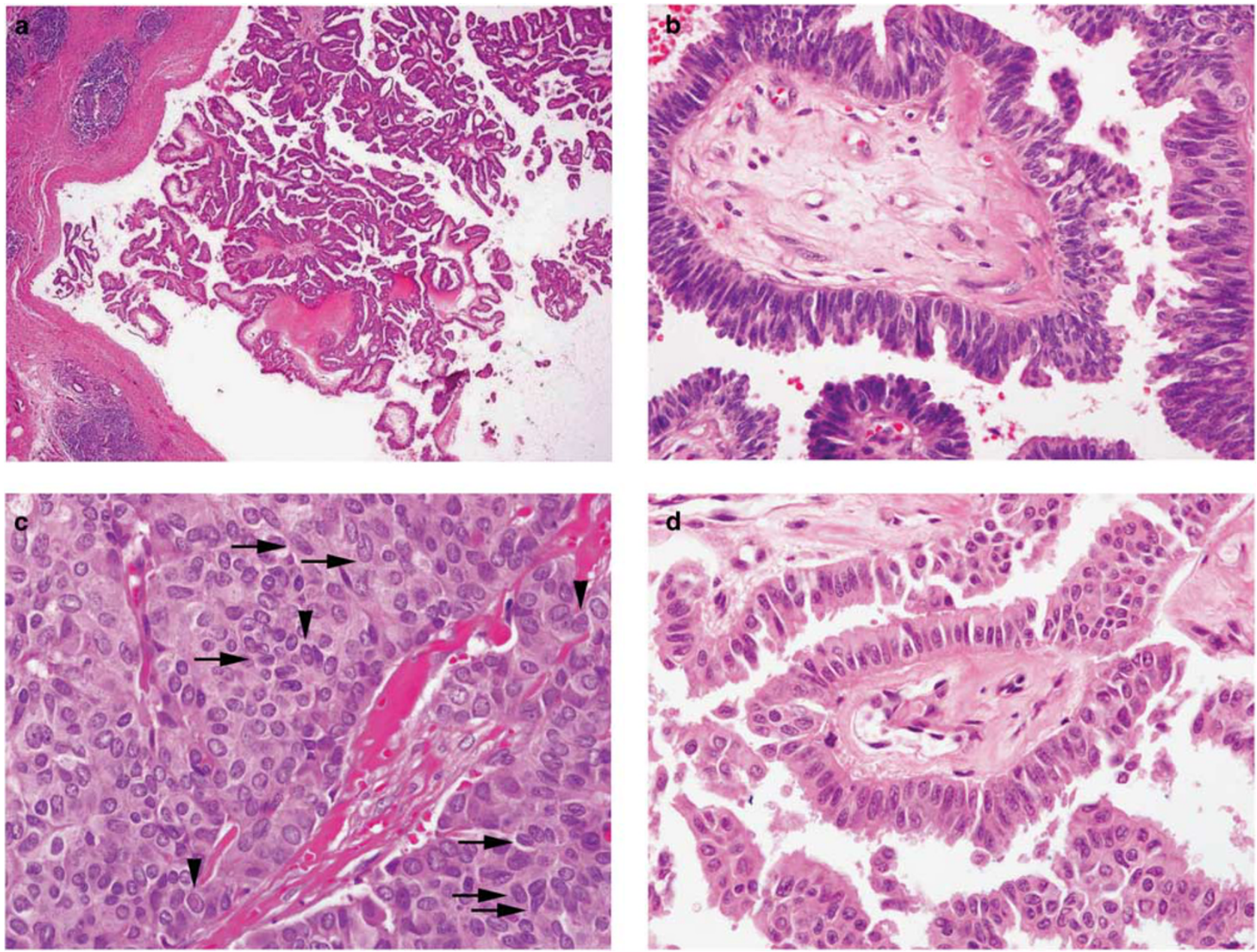

Figure 1 Examples of papillary carcinoma of the breast displaying cytoarchitectural features of papillary thyroid carcinoma. These include a papillary architecture (a), as well as nuclear overlap and stratification (b), nuclear clearing, grooves (arrows) and inclusions (arrowheads) (c), and occasionally, eosinophilic cells with a length at least two times their width (d), resembling the tall-cell variant of papillary thyroid carcinoma. 

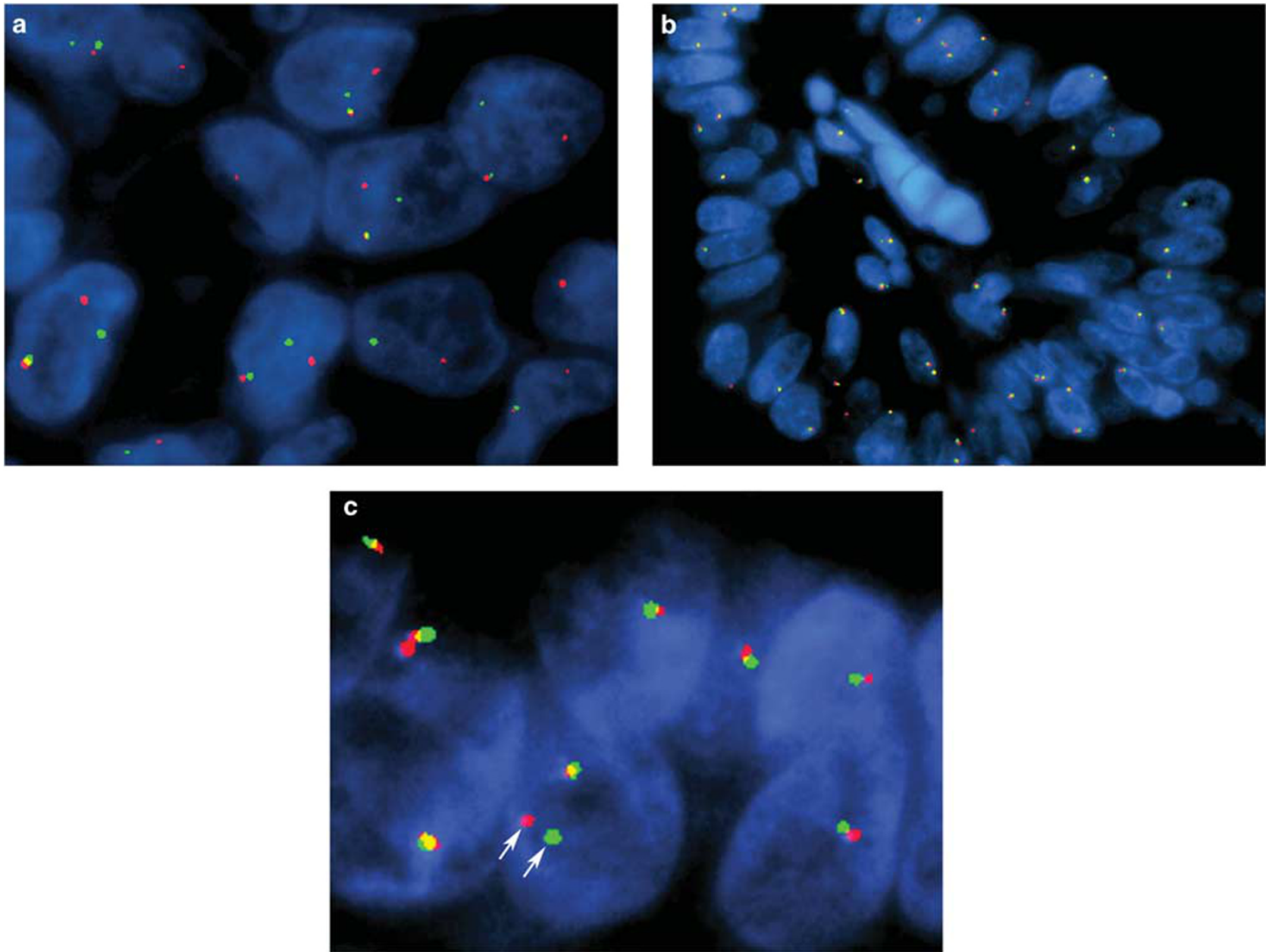

Figure 2 FISH findings in papillary carcinoma of the breast. In the cell line control specimen, the majority of cells showed at least one normally fused yellow or red-green signal and at least one split pair of red and green signals (a). Polyploid cells were also seen with multiple fused and split signals (not shown). In contrast to papillary thyroid carcinoma, most papillary carcinomas of the breast were FISH-negative (b), whereas a few displayed split FISH signals in a small percentage of cells (c), arrows display a pair of split red and green signals) and were considered borderline.

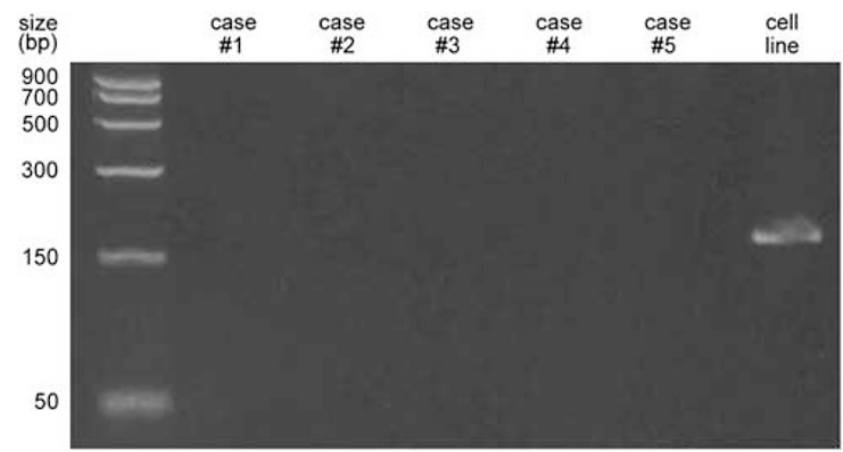

Figure 3 RT-PCR findings in papillary carcinoma of the breast. All tested cases were negative for the RET transcripts evaluated in the study.

was not rearranged and therefore, these breast tumors appear genetically distinct from papillary thyroid carcinoma, suggesting that other genetic or epigenetic changes may account for the appearances seen in papillary carcinomas of the breast.
Papillary thyroid carcinoma is the most common primary thyroid malignancy, accounting for greater than $80 \%$ of thyroid malignancies. ${ }^{10}$ Despite its name, a papillary architecture is not required for diagnosis and instead, this tumor is now defined by the presence of its 'distinctive nuclear features'. ${ }^{10}$ These nuclear features include overlap, grooves, clearing, and inclusions. ${ }^{7,10}$ In recent years it has also become apparent that conventional papillary thyroid carcinoma is characterized by one of two types of genetic abnormalities, chromosomal rearrangements involving either the RET or the NTRK tyrosine kinase proto-oncogenes, or point mutations in the serine-threonine kinase $B R A F$ or in $R A S^{8,9}$

In contrast to papillary thyroid carcinoma, papillary carcinoma of the breast is relatively rare and accounts for less than $2 \%$ of breast carcinomas. ${ }^{17-19}$ Nevertheless, it often displays stratified columnar epithelial cells covering fibrovascular cores $^{1-3}$ and, as such, resembles other papillary tumors, including papillary thyroid carcinoma. In addition, there are 
10 well-documented cases in the literature of breast tumors that at least partially resemble the tall-cell variant of papillary thyroid carcinoma. ${ }^{4-6}$ Eusebi et $a l^{4}$ first described a series of five breast neoplasms all of which displayed solid and papillary areas composed of columnar to cuboidal eosinophilic cells with grooved nuclei; there was variable nuclear clearing and two cases also displayed intranuclear inclusions. Myoepithelial cells were evident in three cases suggesting at least a focal in situ component. In 2006, Cameselle-Teijeiro et $a l^{5}$ reported a very similar breast neoplasm that also displayed in situ and invasive components. Most recently, Tosi et $a l^{6}$ reported four additional cases with identical clinical, morphological, and immunohistochemical findings. The fact that all 10 of these primary breast neoplasms had at least focal papillary areas raises a very interesting question: can the classic cytoarchitectural features of papillary thyroid carcinoma be identified not only in this unique breast neoplasm, but in other papillary carcinomas of the breast as well? One of the goals of our study was to specifically address this question.

Indeed, we found that $79 \%$ of papillary carcinomas of the breast harbored at least one of the cytoarchitectural features of papillary thyroid carcinoma. However, compared with intracystic/encapsulated papillary carcinoma, these features were significantly less prevalent in solid papillary carcinoma, as only one case focally displayed nuclear grooves. Such differences between these two forms of papillary carcinoma of the breast are not surprising given the unique nature of solid papillary carcinoma. $^{20,21}$ It is also interesting that our frequencies of nuclear clearing $(27 \%)$ and nuclear inclusions $(3 \%)$ in papillary carcinoma of the breast, both cytological features that some consider more specific for papillary thyroid carcinoma, ${ }^{7,22,23}$ were considerably less common than nuclear overlap $(73 \%)$ and nuclear grooves $(42 \%)$. This suggests that these latter two features alone are probably not very useful for the diagnosis of papillary thyroid carcinoma outside the confines of the thyroid gland, where one may encounter a large variety of cell types harboring nuclear grooves. ${ }^{24}$ It should be noted at this point that intranuclear inclusions have been previously described in intraductal epithelial proliferations of the breast, ${ }^{25}$ as well as in a case of invasive ductal carcinoma of apocrine type. ${ }^{26}$

The other question we wanted to answer in this study is whether papillary carcinoma of the breast harbors any of the genetic abnormalities characteristic of papillary thyroid carcinoma. To address this issue, we decided to evaluate our cases using a 'twostep' approach. We first used FISH to 'screen' for major structural changes in the RET gene and then followed up with single round and nested RT-PCR to evaluate for the presence or absence of four common $R E T$ gene rearrangements.
Fifteen of the 19 cases tested by FISH were negative, whereas four cases showed split FISH signals. However, the percentage of cells with split signals $(9-11 \%)$ was much lower than that seen in the papillary thyroid carcinoma cell line $(>80 \%)$ and the two papillary carcinoma patient specimens (40 and $50 \%$, respectively). The significance of finding a small percentage of cells in four of our breast cases with split FISH signals is not clear. Although it could be argued that the subset of neoplastic cells with split signals could have acquired RET rearrangements as a secondary genetic alteration, RT-PCR finding (despite its limitationssee next paragraph below) did not support this, as no fusion transcripts were found. Accordingly, we considered the four breast cases to be equivocal or borderline for RET rearrangements. It is also important to consider that the FISH methodology can only detect major structural changes in the $R E T$ gene and cannot exclude the presence of smaller rearrangements such as small inversions within or around the gene.

On the other hand, the RT-PCR results were clear-cut; as all 19 cases with amplifiable RNA tested were negative for the four different RET fusion transcripts evaluated in the study. This finding is similar to that seen in the originally described cases of breast tumor resembling the tallcell variant of papillary thyroid carcinoma, as Eusebi et $\mathrm{I}^{27}$ subsequently evaluated three of these cases for the presence of RET/PTC and RET/PTC3 transcripts and found that all three were negative. Nonetheless, it is noteworthy that our study cannot exclude the presence of the other rare RET fusions, either previously described in the literature or novel.

Another genetic abnormality that we did not test for in our cases is point mutations in the $B R A F$ gene; by some accounts, ${ }^{8}$ this is the most common alteration in papillary thyroid carcinoma. Interestingly, the single breast tumor resembling the tall-cell variant of papillary thyroid carcinoma that has been previously tested failed to show any mutation after sequencing of exon 15 of $B R A F^{5}$ Similarly, a recent study of various papillary lesions of the breast published only in abstract form,$^{28}$ showed that all papillary lesions tested, including six papillomas, one atypical papilloma, three cases of papillary ductal carcinoma in situ, and three intracystic papillary carcinomas, lacked $B R A F$, as well as NRAS and KRAS mutations. A novel finding however, was the identification of an identical point mutation in codon 61 of the HRAS gene in two of the intracystic papillary carcinomas and not in any of the other lesions. Clearly, more work is needed to determine the prevalence of HRAS mutations and whether they are associated with any particular morphological features.

In summary, we have shown that papillary carcinomas of the breast, not infrequently, display one or more of the cytoarchitectural features 
characteristic of papillary thyroid carcinoma. Our findings also suggest that the presence of these features in such carcinomas are not secondary to common RET rearrangements. Additional studies are needed to identify the genetic or epigenetic changes, if any, that may be pathogenetically related to these morphological appearances.

\section{Disclosure/conflict of interest}

None declared.

\section{References}

1 Mulligan AM, O’Malley FP. Papillary lesions of the breast: a review. Adv Anat Pathol 2007;14:108-119.

2 Collins LC, Schnitt SJ. Papillary lesions of the breast: selected diagnostic and management issues. Histopathology 2008;52:20-29.

3 Schnitt SJ, Collins LC. Papillary lesions. In: Biopsy Interpretation of the Breast. Wolters Kluwer/Lippincott Williams \& Wilkins: Philadelphia, 2008, pp 205-235.

4 Eusebi V, Damiani S, Ellis IO, et al. Breast tumor resembling the tall cell variant of papillary thyroid carcinoma: report of 5 cases. Am J Surg Pathol 2003;27:1114-1118.

5 Cameselle-Teijeiro J, Abdulkader I, Barreiro-Morandeira $\mathrm{F}$, et al. Breast tumor resembling the tall cell variant of papillary thyroid carcinoma: a case report. Int J Surg Pathol 2006;14:79-84.

6 Tosi AL, Ragazzi M, Asioli S, et al. Breast tumor resembling the tall cell variant of papillary thyroid carcinoma: report of 4 cases with evidence of malignant potential. Int J Surg Pathol 2007;15:14-19.

7 Vickery Jr AL, Carcangiu ML, Johannessen JV, et al. Papillary carcinoma. Semin Diagn Pathol 1985;2:90-100.

8 DeLellis RA. Pathology and genetics of thyroid carcinoma. J Surg Oncol 2006;94:662-669.

9 Pfeifer JD. Endocrine System. In: Pfeifer JD (ed). Molecular Genetic Testing in Surgical Pathology. Lippincott Williams and Wilkens: Philadelphia, 2006, pp 232-249.

10 DeLellis RA. Pathology and Genetics of Tumours of Endocrine Organs. IARC Press: Lyon, 2004, 320 pp.

11 Ghossein R, Livolsi VA. Papillary thyroid carcinoma tall cell variant. Thyroid 2008;18:1179-1181.

12 Bridge RS, Rajaram V, Dehner LP, et al. Molecular diagnosis of Ewing sarcoma/primitive neuroectodermal tumor in routinely processed tissue: a comparison of two FISH strategies and RT-PCR in malignant round cell tumors. Mod Pathol 2006;19:1-8.

13 Basolo F, Giannini R, Toniolo A, et al. Establishment of a non-tumorigenic papillary thyroid cell line (FB-2) carrying the RET/PTC1 rearrangement. Int J Cancer 2002;97:608-614.
14 O’Sullivan MJ, Perlman EJ, Furman J, et al. Visceral primitive peripheral neuroectodermal tumors: a clinicopathologic and molecular study. Hum Pathol 2001;32:1109-1115.

15 Liu RT, Chou FF, Wang CH, et al. Low prevalence of RET rearrangements (RET/PTC1, RET/PTC2, RET/ PTC3, and ELKS-RET) in sporadic papillary thyroid carcinomas in Taiwan Chinese. Thyroid 2005;15: 326-335.

16 Ferrari A, Casanova M, Bisogno G, et al. Clear cell sarcoma of tendons and aponeuroses in pediatric patients: a report from the Italian and German Soft Tissue Sarcoma Cooperative Group. Cancer 2002;94:3269-3276.

17 Carter D. Intraductal papillary tumors of the breast: a study of 78 cases. Cancer 1977;39:1689-1692.

18 Fisher ER, Redmond C, Fisher B. Pathologic findings from the National Surgical Adjuvant Breast Project (Protocol no. 4). VI. Discriminants for five-year treatment failure. Cancer 1980;46: 908-918.

19 Framarino dei Malatesta ML, Piccioni MG, Felici A, et al. Intracystic carcinoma of the breast. Our experience. Eur J Gynaecol Oncol 1992;13:40-44.

20 Maluf HM, Koerner FC. Solid papillary carcinoma of the breast. A form of intraductal carcinoma with endocrine differentiation frequently associated with mucinous carcinoma. Am J Surg Pathol 1995;19: 1237-1244.

21 Nassar H, Qureshi H, Volkanadsay N, et al. Clinicopathologic analysis of solid papillary carcinoma of the breast and associated invasive carcinomas. Am J Surg Pathol 2006;30:501-507.

22 Lloyd RV, Erickson LA, Casey MB, et al. Observer variation in the diagnosis of follicular variant of papillary thyroid carcinoma. Am J Surg Pathol 2004;28:1336-1340.

23 Rosai J, Zampi G, Carcangiu ML. Papillary carcinoma of the thyroid. A discussion of its several morphologic expressions, with particular emphasis on the follicular variant. Am J Surg Pathol 1983;7:809-817.

24 Batistatou A, Scopa C. Pathogenesis and diagnostic significance of nuclear grooves in thyroid and other sites. Int J Surg Pathol 2009;17:107-110.

25 Tavassoli FA, Majeste RM, Snyder RC. Intranuclear helioid inclusions in mammary intraductal hyperplasias. Ultrastruct Pathol 1991;15:267-279.

26 Grekou AN, Stravoravdi P, Patakiouta F, et al. Intranuclear helioid inclusions in a case of breast carcinoma. Breast J 1999;5:63-64.

27 Eusebi V, Tallini G, Rosai J. Nuclear alterations and RET/PTC activation. Am J Surg Pathol 2004;28: 974-975.

28 Nicosia Esposito N, Nikiforova MN, Nikiforov YE, et al. Mutational profile of RAS and BRAF genes in papillary lesions of the breast: identification of a common HRAS mutation in intracystic papillary carcinoma. Mod Pathol 2008;21:47A. 